\title{
Principles of vortex light generation from electronically excited nanoscale arrays
}

\author{
Mathew D. Williams, David S. Bradshaw, David L. Andrews* \\ School of Chemistry, University of East Anglia, Norwich Research Park, Norwich NR4 7TJ, U. K.
}

\begin{abstract}
It has recently been shown possible to directly generate an optical vortex (a beam of light endowed with orbital angular momentum) by spontaneous emission from a molecular exciton array. This contrasts with most established methods, which typically rely on the modification of a conventional beam by an appropriate optical element (for example, a $q$-plate) to impose the requisite helical twist of a vortex. The new procedure is achieved by nanofabricating a chiral arrangement of chromophores into a ring of specifically configured symmetry, supporting a doubly degenerate (conjugated) exciton with the appropriate azimuthal phase progression. It emerges that the symmetry elements present in the phase structure of the optical field, produced by emission from these degenerate excitons on a array, exhibits precisely the sought character of an optical vortex. The highest order of exciton symmetry, including the corresponding splitting of the electronic states, dictates the maximum magnitude of the topological charge. Work is now progressing on computer simulations aiming to reveal the detailed pattern of polarization behaviour in the emitted light, in which the vector character of the beam progresses around the phase singularity along the beam propagation axis. Significantly, this analysis points to the emission of radiation with polarization varying over the beam profile.
\end{abstract}

Keywords: optical angular momentum, Laguerre-Gaussian, optical vortices, quantum optics, chirality, structured light.

\section{INTRODUCTION}

The field of optics has been enhanced by the rapid development of research involving laser light endowed with orbital angular momenta (OAM). These structures are known as optical vortices, and their angular momentum properties are completely distinct and separable from those associated with photon spin. ${ }^{1}$ The structure of such vortex beams is primarily measured by a topological charge, $l$ (signifying an OAM of $l \hbar$ per photon), with positive or negative parity denoting either left- or right- handed gyration respectively. There is an increasing recognition of the wide scope to exploit the unique properties of optical vortex beams, with wide-ranging applications. ${ }^{2-4}$ Within the past year, we have offered a novel theoretical means to generate an optical vortex beam that contrasts to conventional methods requiring the modification of a pre-existing beam. ${ }^{5-7}$ Here the model, involving spontaneous light emission, is fully elucidated and our latest findings on this topic are presented.

\section{EMISSION FROM ARRAY STRUCTURES}

\subsection{OAM values of the generated beam}

We begin with a brief summary of the mechanism for the direct generation of an optical vortex. The symmetry constraints on the emitter array are governed by certain fundamental principles. In physical form, the structure is to be constructed in the form of a ring-like array in which the centers of each molecular component lie in a single plane, as visualized in Figure 1. To secure the necessary conditions for emissive decay, a phase progression is applied around the ring; such forms of electronic excitation will form spontaneously, given arrays of the correct symmetry type. The detailed phase property is determined by the character of each specific excited state, rather than the array upon which that state resides. This signifies that, given an array that has a sufficient number of components, it is possible for vortex 


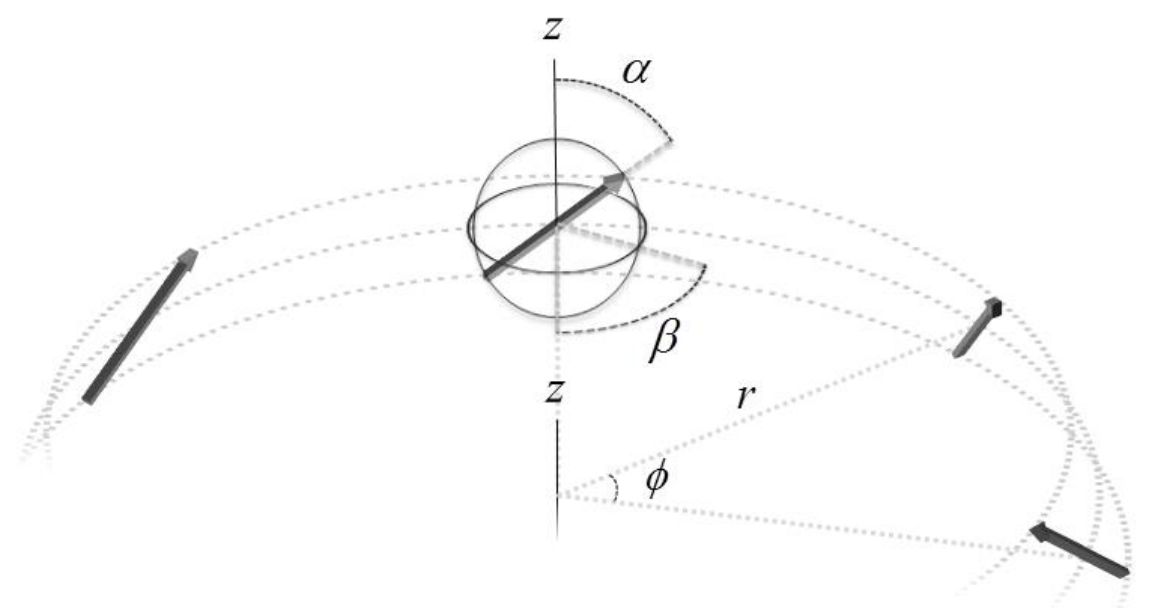

Figure 1. One potential schematic cross-section for a $n$-dipole nanoarray conforming to point group $C_{n}$, where each emitter has a circular disposition around the ring represented by $(r, \theta, z)$ and orientation $(\alpha, \beta, z)$.

structures of various topological charge to be generated from a single array. Suitable symmetry structures required for the arrays can be identified by examining the Schoenflies point group tables. ${ }^{8-9}$ Point group symmetries that feature biaxial degeneracy are required (for the two axes defining the plane of the ring) with a lack of 'vertical' mirror symmetry (whose presence would undermine the sought progression of phase around the ring). Satisfying these criteria, suitable families of point groups prove to be $C_{n}, C_{n}, S_{n}, T, T_{h}$, where $n$ is the number of chromophores comprising the ring; these are all symmetry groups in which the majority of allowed symmetry elements are pure rotations. Attention now focuses on the doubly degenerate irreducible representations that are found within these groups. On excitation of the ring-like array the electronic excitation is delocalized across the composite molecules to form an exciton, hence, our concern is with decay transitions from those exciton states. ${ }^{10}$ Assuming that such decay terminates at a ground state (usually a totally symmetric representation), then the symmetry character of the initial excited state maps directly on to the symmetry of the decay transition, and hence the vortex structure of the emitted electromagnetic radiation. Table 1 details the possible integer values of orbital angular momentum that may be emitted from an array with the defined symmetry below, characterized by its respective Schoenflies point group. An analysis on the quantum theory behind our model, including information on the excitonic wavefunction and Hamiltonian, is to be found in another recent Proceedings.

Cylindrical polar coordinates $(r, \theta, z)$ are logically deployed in the analysis for the ring, whereas the individual dipoles are better described by a spherical polar coordinate basis $(\alpha, \beta, z)$. The dipole network displayed in Fig. 1 is representative of the symmetry requirements for a $C_{n}$ point group structure. Setting $\alpha=\pi / 2$, the array adopts a higher order of symmetry and thus group, $C_{n h}$, which is also a permitted structure. However, if $\alpha \neq 0, \beta=0$ or $\pi$, we form a structure that is no longer capable of supporting an appropriate excitonic state, in consequence of the vertical mirror plane introduced, which undermines the spiral azimuthal phase structure required for a vortex emission.

\subsection{Structure of the excitonic energy levels}

As a result of their differences in symmetry, the energy levels that relate to the exciton will split. Taking the simplest case of $n=3$ emitters, noting that a two-molecule array will not produce the desired effect, it is determined that the energy levels correspond to a non-degenerate state (belonging to the totally symmetric representation $A$ ), with energy $E_{u}-2 U$, and two doubly degenerate ( $E$ representation) states of energy $E_{u}+U$ - where $E_{u}$ is the energy of the isolated molecular component and $U$ relates to the coupling between the components. Table 2 shows information corresponding to an $n$ component array; here, the integer $p$ is assigned to each permitted excitonic symmetry and $q$ is used to label each doubly degenerate energy level of the exciton. From Table 2, an energy level diagram is now constructed for the $n=9$ component case and displayed in Figure 2. ${ }^{11}$ 
Table 1: Summary of the allowed topological charge $l$, for OAM outputs based on arrays of the allowed symmetry groups. For the $S_{n}$ groups, $q=0$ if $i \notin\left\{S_{n}\right\} ; q=1$ if $i \in\left\{S_{n}\right\}$. In addition (not shown), the groups $T$ and $T_{h}$ also support a unit topological charge. The entries in the last row, which apply to the general case, express the necessary conditions incorporating a floor function ${ }^{12}$.

\begin{tabular}{|l|l|l|}
\hline \multirow{2}{*}{$\begin{array}{l}\text { Number } \\
\text { of } \\
\text { emitters }\end{array}$} & \multicolumn{3}{|l|}{ Symmetry group } & \multicolumn{1}{l|}{$S_{n}$} \\
\cline { 2 - 3 }$C_{n}, C_{n h}$ & - \\
\hline 3 & 1 & 1 \\
\hline 4 & 1 & - \\
\hline 5 & 1,2 & 1 \\
\hline 6 & 1,2 & - \\
\hline 7 & $1,2,3$ & $1,2,3$ \\
\hline 8 & $1,2,3$ & - \\
\hline 9 & $1,2,3,4$ & 1,2 \\
\hline 10 & $1,2,3,4$ & - \\
\hline 11 & $1,2,3,4,5$ & $1,2,3,4,5$ \\
\hline 12 & $1,2,3,4,5$ & $|l| \leq\left\lfloor\left(\frac{1}{2}\right)^{q} \frac{n-2}{2}\right]$ \\
\hline$n$ & $\left.|l| \leq \leq \frac{n-1}{2}\right]$ & \\
\hline
\end{tabular}

Table 2: The irreducible representations (Irrep) of the $C_{n}$ excited states for $n=\{3: 9\}$

\begin{tabular}{|c|c|c|c|c|c|c|c|c|c|c|}
\hline$n$ & $p$ & 1 & 2 & 3 & 4 & 5 & 6 & 7 & 8 & 9 \\
\hline \multirow{2}{*}{3} & $q$ & 1 & -1 & 0 & & & & & & \\
\hline & Irrep & $E_{1}$ & $E_{1}$ & $A$ & & & & & & \\
\hline \multirow{2}{*}{4} & $q$ & 1 & 2 & -1 & 0 & & & & & \\
\hline & Irrep & $E_{1}$ & $B$ & $E_{1}$ & $A$ & & & & & \\
\hline \multirow{2}{*}{5} & $q$ & 1 & 2 & -2 & -1 & 0 & & & & \\
\cline { 2 - 12 } & Irrep & $E_{1}$ & $E_{2}$ & $E_{2}$ & $E_{1}$ & $A$ & & & & \\
\hline \multirow{2}{*}{6} & $q$ & 1 & 2 & 3 & -2 & -1 & 0 & & & \\
\cline { 2 - 12 } & Irrep & $E_{1}$ & $E_{2}$ & $B$ & $E_{2}$ & $E_{1}$ & $A$ & & & \\
\hline \multirow{2}{*}{7} & $q$ & 1 & 2 & 3 & -3 & -2 & -1 & 0 & & \\
\cline { 2 - 12 } & Irrep & $E_{1}$ & $E_{2}$ & $E_{3}$ & $E_{3}$ & $E_{2}$ & $E_{1}$ & $A$ & & \\
\hline \multirow{2}{*}{8} & $q$ & 1 & 2 & 3 & 4 & -3 & -2 & -1 & 0 & \\
\hline & Irrep & $E_{1}$ & $E_{2}$ & $E_{3}$ & $E_{4}$ & $E_{3}$ & $E_{2}$ & $E_{1}$ & $A$ & \\
\hline \multirow{2}{*}{9} & $q$ & 1 & 2 & 3 & 4 & -4 & -3 & -2 & -1 & 0 \\
\hline & Irrep & $E_{1}$ & $E_{2}$ & $E_{3}$ & $E_{4}$ & $E_{4}$ & $E_{3}$ & $E_{2}$ & $E_{1}$ & $A$ \\
\hline
\end{tabular}

With the labeling given by the irreducible representations seen in Table 2, the $E_{q}, A$ (and also $B$, if present) states each have a different symmetry energy, as seen in Figure 2. Each doubly degenerate exciton denoted by a subscript $q$ will therefore have a unique and discernable emission wavelength. This is consistent with Kasha's rule (more often applied in the context of vibrational sub-levels), where the emission usually relates to the energy gap between the lowest-lying energy level and the ground state. Physically, the exciton pair with the lowest energy will be favored and associated with the highest $|q|$ value, which will also correspond to the largest topological charge for the emitted vortex. 


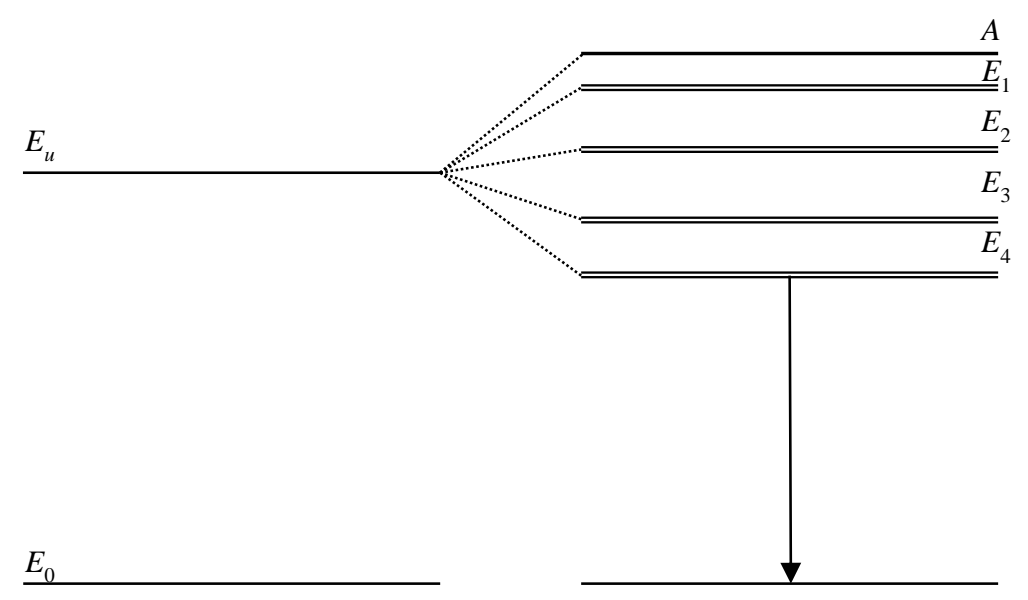

Figure 2: The corresponding excitonic irreducible representations and Davydov energy level splitting for an array of $n=9$ and thus $C_{9}$ point group symmetry as detailed in Table 2.

\section{POLARIZATION STRUCTURES}

In this Section we present preliminary simulations for the phase and amplitude, and also the polarization structure, of electromagnetic fields produced from arrays of the kind detailed above, taking as an example the case of three emitters. These plots represent the vector field established by the exciton, typical of the $l=1$ topological charge the system is tailored for. The left-hand panel of Figure 3 exhibits the phase progression, coded through the colors of the visible spectrum, with a depth of color indicating the field amplitude. In the right-hand panel, arrows indicate the corresponding direction of a linear polarization vector. The observable $\pi$ rotation in the polarization contours is consistent with earlier geometric surface work on umbilic points, ${ }^{13-15}$ and in particular the lemon structure of the singular value in the optical field. ${ }^{16-17}$
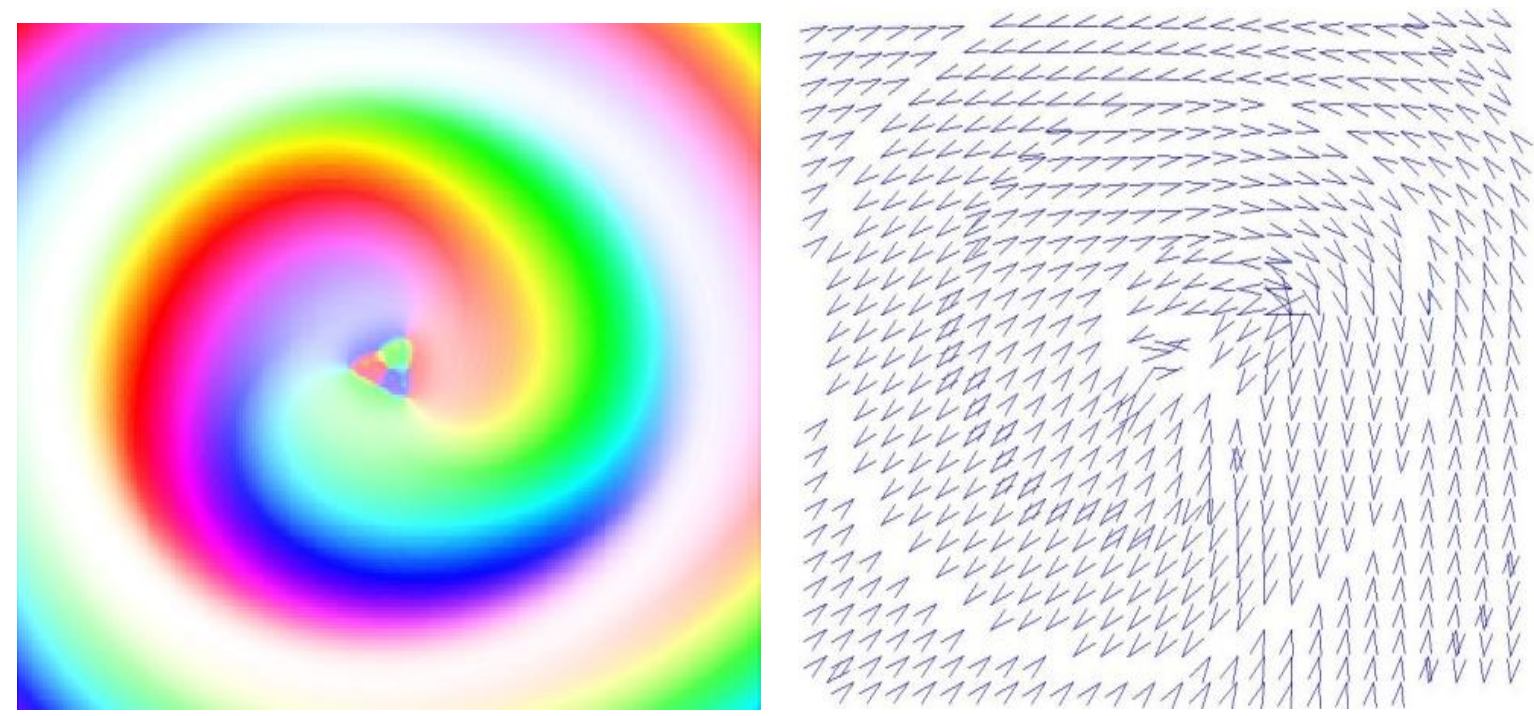

Figure 3: Two depictions for an array of $n=3$ and $l=1$ : a phase map exhibiting the spiral structure of established by the relaxation of the exciton with the amplitude of the overlaid (left); a polarization map indicating the umbilic point coinciding with the singular value in the electric field (right). Plots span $x$ and $y$ distances of to $(0.4 / 2 \pi)$ times the optical wavelength. 


\section{DISCUSSION}

This paper assesses and further develops the possibility that structured light may be produced spontaneously at the single photon level. To generate such twisted light, the analysis describes a ring-like array that needs to be suitably fabricated. In fact, on extension of this groundwork, a variety of possible implementation techniques might be envisaged. An obvious alternative is a multi-chromophore architecture, which could satisfy the necessary conditions if distinct electronic transitions occur in each chromophore; ${ }^{18-19}$ such possibilities could offer means to achieve the symmetry exhibited by Figure 1. By more extensive means, ${ }^{20-21}$ it is conceivable that tailored arrays could be manufactured directly, to provide for extensive electronic couplings between pairs of neighboring nanoscale antennae. There are options for a variety of planar deposition techniques, ${ }^{22-23}$ including those that can produce layered chiral thin films of dielectric materials, ${ }^{24}$ or others that provide for tailored metallic structures exhibiting delocalized electronic motions. ${ }^{25-26}$ Electron beam and other forms of lithography could be used to etch suitable substrate surfaces, ${ }^{27-29}$ and further opportunities might be afforded by quantum dot nanoarrays, ${ }^{30}$ given the possibility of forming anisotropic components with directional emission properties. ${ }^{31}$ Lastly, since magnetic guidance proves amenable to maneuvering magnetizable nanoparticles, this too might afford a means of fulfilling the demanding symmetry conditions without having to fabricate materials with the necessary intrinsic geometry. ${ }^{32}$

\section{ACKNOWLEDGEMENTS}

The authors would like to thank the University of East Anglia and Leverhulme Trust for funding this research.

\section{REFERENCES}

[1] Bialynicki-Birula, I. and Bialynicka-Birula, Z., "Canonical separation of angular momentum of light into its orbital and spin parts,” J. Opt. 13, 064014 (2011).

[2] Allen, L., Barnett, S. M. and Padgett, M. J., [Optical Angular Momentum], Taylor \& Francis Group, Bristol (2003).

[3] Andrews, D. L., [Structured Light and its Applications: An Introduction to Phase-Structured Beams and Nanoscale Optical Forces], Academic, Amsterdam, Boston (2008).

[4] Andrews, D. L. and Babiker, M., [The Angular Momentum of Light], Cambridge University Press, Cambridge, UK (2013).

[5] Coles, M. M., Williams, M. D., Saadi, K., Bradshaw, D. S. and Andrews, D. L., "Chiral nanoemitter array: A launchpad for optical vortices,” Laser \& Photon. Rev. 7, 1088-1092 (2013).

[6] Williams, M. D., Coles, M. M., Saadi, K., Bradshaw, D. S. and Andrews, D. L., “Optical Vortex Generation from Molecular Chromophore Arrays," Phys. Rev. Lett. 111, 153603 (2013).

[7] Williams, M. D., Coles, M. M., Bradshaw, D. S. and Andrews, D. L., "Optical vortex mode generation by nanoarrays with a tailored geometry," Proc. SPIE 8999, 89990Q (2014).

[8] Salthouse, J. A., Ware, M. J. and Ware, M. J., [Point Group Character Tables and Related Data], Cambridge University Press, London (1972).

[9] Carter, R. L., [Molecular symmetry and group theory], J. Wiley, New York (1998).

[10] Valkunas, L. A., Darius and Mancal, T., [Molecular excitation dynamics and relaxation: quantum theory and spectroscopy], Wiley-VCH, Weinheim (2013).

[11] Williams, M. D., Coles, M. M., Bradshaw, D. S. and Andrews, D. L., "Direct generation of optical vortices," Phys. Rev. A 89, 033837 (2014).

[12] Weisstein, E. W., [CRC Concise Encyclopedia of Mathematics], Chapman \& Hall/CRC, Boca Raton (2003).

[13] Berry, M. and Hannay, J., "Umbilic points on Gaussian random surfaces," J. Phys. A: Math. Gen. 10 , 1809 (1977).

[14] Dennis, M. R., “Polarization singularity anisotropy: determining monstardom,” Opt. Lett. 33, 2572-2574 (2008).

[15] Wolf, E., [Progress in Optics], Elsevier Science, Amsterdam (2009). 
[16] Galvez, E. J., Rojec, B. L. and Beach, K., "Mapping of all polarization-singularity C-point morphologies,” Proc. SPIE 8999, 89990I (2014).

[17] Kumar, V. and Viswanathan, N. K., "Topological aspects of polarization structured beams," Proc. SPIE 8999, 89990M (2014).

[18] Brunel, J., Mongin, O., Jutand, A., Ledoux, I., Zyss, J. and Blanchard-Desce, M., "Propeller-Shaped Octupolar Molecules Derived from Triphenylbenzene for Nonlinear Optics: Synthesis and Optical Studies," Chem. Mater. 15, 4139-4148 (2003).

[19] Rouxel, C., Le Droumaguet, C., Macé, Y., Clift, S., Mongin, O., Magnier, E. and Blanchard-Desce, M., "Octupolar Derivatives Functionalized with Superacceptor Peripheral Groups: Synthesis and Evaluation of the Electron-Withdrawing Ability of Potent Unusual Groups," Chem. Eur. J. 18, 12487-12497 (2012).

[20] Barth, J. V., Costantini, G. and Kern, K., "Engineering atomic and molecular nanostructures at surfaces," Nature 437, 671-679 (2005).

[21] Gates, B. D., Xu, Q. B., Stewart, M., Ryan, D., Willson, C. G. and Whitesides, G. M., "New approaches to nanofabrication: Molding, printing, and other techniques," Chem. Rev. 105, 1171-1196 (2005).

[22] Knez, M., Nielsch, K. and Niinistö, L., "Synthesis and Surface Engineering of Complex Nanostructures by Atomic Layer Deposition," Adv. Mater. 19, 3425-3438 (2007).

[23] van Dorp, W. F. and Hagen, C. W., "A critical literature review of focused electron beam induced deposition,” J. Appl. Phys. 104, 081301 (2008).

[24] Hodgkinson, I. J., Lakhtakia, A., Wu, Q. H., De Silva, L. and McCall, M. W., “Ambichiral, equichiral and finely chiral layered structures," Opt. Commun. 239, 353-358 (2004).

[25] Papakostas, A., Potts, A., Bagnall, D. M., Prosvirnin, S. L., Coles, H. J. and Zheludev, N. I., "Optical Manifestations of Planar Chirality," Phys. Rev. Lett. 90, 107404 (2003).

[26] Plum, E., Zhou, J., Dong, J., Fedotov, V. A., Koschny, T., Soukoulis, C. M. and Zheludev, N. I., "Metamaterial with negative index due to chirality," Phys. Rev. B 79, 035407 (2009).

[27] Watt, F., Bettiol, A. A., Van Kan, J. A., Teo, E. J. and Breese, M. B. H., "Ion beam lithography and nanofabrication: A review," Int. J. Nanosci. 04, 269-286 (2005).

[28] Guo, L. J., "Nanoimprint lithography: Methods and material requirements," Adv. Mater. 19, 495-513 (2007).

[29] Xie, Z., Yu, W., Wang, T., Zhang, H., Fu, Y., Liu, H., Li, F., Lu, Z. and Sun, Q., "Plasmonic Nanolithography: A Review," Plasmonics 6, 565-580 (2011).

[30] Abramson, J., Palma, M., Wind, S. J. and Hone, J., "Quantum dot nanoarrays: Self-assembly with single-particle control and resolution," Adv. Mater. 24, 2207-2211 (2012).

[31] Lundskog, A., Hsu, C.-W., Fredrik Karlsson, K., Amloy, S., Nilsson, D., Forsberg, U., Olof Holtz, P. and Janzen, E., "Direct generation of linearly polarized photon emission with designated orientations from site-controlled InGaN quantum dots," Light Sci. Appl. 3, e139 (2014).

[32] Demirörs, A. F., Pillai, P. P., Kowalczyk, B. and Grzybowski, B. A., "Colloidal assembly directed by virtual magnetic moulds," Nature 503, 99-103 (2013).

\footnotetext{
*david.andrews@physics.org
} 